\title{
Physical Activity and Quality of Life in Severely Obese Adults during a Two-Year Lifestyle Intervention Programme
}

\author{
Randi Jepsen, ${ }^{1,2}$ Eivind Aadland, ${ }^{1}$ Lesley Robertson, ${ }^{3}$ Ronette L. Kolotkin, ${ }^{1,4,5,6,7}$ \\ John Roger Andersen, ${ }^{1,6}$ and Gerd Karin Natvig ${ }^{2}$ \\ ${ }^{1}$ Faculty of Health Studies, Sogn og Fjordane University College, P.O. Box 523, 6803 Førde, Norway \\ ${ }^{2}$ Department of Global Public Health and Primary Care, University of Bergen, 5020 Bergen, Norway \\ ${ }^{3}$ Red Cross Haugland Rehabilitation Centre, 6968 Flekke, Norway \\ ${ }^{4}$ Quality of Life Consulting, Durham, NC 27705, USA \\ ${ }^{5}$ Department of Community and Family Medicine, Duke University School of Medicine, Durham, NC 27708, USA \\ ${ }^{6}$ Department of Surgery, Førde Central Hospital, 6807 Førde, Norway \\ ${ }^{7}$ Morbid Obesity Centre, Vestfold Hospital Trust, 3103 Tønsberg, Norway \\ Correspondence should be addressed to Randi Jepsen; randi.jepsen@hisf.no
}

Received 19 August 2014; Accepted 23 December 2014

Academic Editor: R. Prager

Copyright (C) 2015 Randi Jepsen et al. This is an open access article distributed under the Creative Commons Attribution License, which permits unrestricted use, distribution, and reproduction in any medium, provided the original work is properly cited.

It is unknown how changes in physical activity may affect changes in quality of life (QoL) outcomes during lifestyle interventions for severely obese adults. The purpose of this study was to examine associations in the patterns of change between objectively assessed physical activity as the independent variable and physical, mental, and obesity-specific QoL and life satisfaction as the dependent variables during a two-year lifestyle intervention. Forty-nine severely obese adults (37 women; $43.6 \pm 9.4$ years; body mass index $42.1 \pm 6.0 \mathrm{~kg} / \mathrm{m}^{2}$ ) participated in the study. Assessments were conducted four times using Medical Outcomes Study Short-Form 36 Health Survey (SF-36), Obesity-Related Problems (OP) scale, a single item on life satisfaction, and accelerometers. The physical component summary (PCS) score and the mental component summary (MCS) score were used as SF-36 outcomes. Associations were determined using linear regression analyses and reported as standardized coefficients (stand. coeff.). Change in physical activity was independently associated with change in PCS (stand. coeff. $=0.35, P=.033$ ), MCS (stand. coeff. $=0.51$, $P=.001$ ), OP (stand. coeff. $=-0.31, P=.018$ ), and life satisfaction (stand. coeff. $=0.39, P=.004$ ) after adjustment for gender, age, and change in body mass index.

\section{Introduction}

Severely obese adults seeking lifestyle interventions report impaired physical, mental, and obesity-specific quality of life (QoL) [1-3]. Thus, several studies have included QoL as a primary outcome in evaluation of multicomponent lifestyle interventions for these individuals [4-7]. These studies have proposed physical activity to be a contributor to unexplained improvements in QoL [4-7]. Danielsen et al. [4] and Karlsen et al. [5] demonstrated improvements in the physical component summary (PCS) score and mental component summary (MCS) score of the Medical Outcomes Study ShortForm 36 Health Survey (SF-36) at the end of one-year, partly residential interventions. Blissmer et al. [7] reported similar findings after a six-month, outpatient intervention for overweight to obese adults. Repeated measures in two of these studies revealed improvements in both PCS and MCS after an initial, intensive intervention phase whereas longer-term maintenance varied $[4,7]$. The results reported by Blissmer et al. [7] were independent of weight loss while Danielsen el al. [4] revealed positive associations between weight loss and improvements in PCS but not MCS. With regard to obesity-specific QoL, the Swedish Obese Subjects (SOS) study developed and used the instrument ObesityRelated Problems (OP) scale. After four [2] and ten years [8], the authors found significant improvements in severely obese adults who had received "conventional treatment." However, the treatment was not standardised but was provided in 
accordance with local routines by many primary health care centres. Improvements in OP were positively correlated with weight loss $[2,8]$. In contrast, despite weight regain, Kaukua et al. [6] demonstrated improvements in OP at the end of a two-year follow-up of severely obese individuals who completed a four-month, outpatient intervention.

Compared to physical, mental, and obesity-specific QoL, life satisfaction is a broader QoL construct representing a subjective and global assessment of all major dimensions of life [9]. Using single item measures on life satisfaction, obesity was associated with impaired life satisfaction in two U.S. population studies $[10,11]$, whereas a Danish epidemiological study, controlling for a cluster of lifestyle-related factors including body mass index (BMI), found independent positive associations between self-reported physical activity and life satisfaction [12].

Multicomponent lifestyle interventions for severe obesity aim for a sustainable change of behaviour related to diet and physical activity [13]. Physical activity is beneficial for body composition and fitness in obese individuals undergoing dietary energy restriction [14] and reduces adverse cardiovascular outcomes of obesity $[15,16]$. In the eight-year follow-up of the Look AHEAD study, self-reported physical activity was associated with initial and maintained weight loss in overweight to obese subjects with type 2 diabetes [17]. Cross-sectional, unadjusted analyses in studies on treatmentseeking severely obese adults have demonstrated positive correlations between self-reported physical activity and PCS $[18,19]$ and MCS [18]. We found positive independent associations between objectively assessed physical activity and life satisfaction prior to a lifestyle intervention for severely obese adults [20] and did a series of studies on patterns of change in the participants during the intervention. We used accelerometers to objectively measure physical activity, collected data at multiple time points, and found positive associations in the patterns of change between physical activity and aerobic fitness [21], fat mass [22], and lipoproteins [23], confirming the importance of physical activity for clinical and anthropometric outcomes in lifestyle interventions. To our knowledge, a similar design has not been used to examine associations between change in physical activity and QoL outcomes over time. Therefore, the present study examines associations between change in objectively assessed physical activity as the independent variable and change in physical, mental, and obesity-specific QoL and life satisfaction as the dependent variables during a two-year, multicomponent lifestyle intervention.

\section{Design and Methods}

This study is part of the Haugland Obesity Study, a prospective cohort study on severely obese adults who participated in a publicly funded two-year lifestyle intervention at Red Cross Haugland Rehabilitation Centre (RCHRC) in Western Norway. Data were collected between February 2010 and October 2012 and the present study used data from four time points: baseline prior to the intervention $(T 0)$, six weeks later, at the end of the first residential stay, $(T 1)$, and prior to the residential stays one (T2) and two (T3) years from baseline.
Referral of patients was done by general practitioners in accordance with the right to admission to the Norwegian specialist health services (i.e., BMI $\geq 40 \mathrm{~kg} / \mathrm{m}^{2}$ or $\geq 35 \mathrm{~kg} / \mathrm{m}^{2}$ with comorbidities) [24]. In total, 53 eligible patients from the age of 18 to 60 years, divided into four groups, had their first residential stay between February 2010 and October 2011. Exclusion criteria included previous obesity surgery or referral to obesity surgery; severe cardiovascular disease; pregnancy; substance or alcohol abuse; and impaired physical functioning or mental problems which could interfere with adherence to the intervention.

2.1. Intervention. The intervention has been described in detail previously [25]. Briefly, the patients spent a total of 15 weeks at RCHRC divided into four stays of six, three (after three months), three (at year one), and three (at year two) weeks. A multiprofessional team managed the intervention. The overall goal was to improve the QoL of the patients, while weight loss, improved mental health and physical fitness, and reduction of obesity-related medical problems served as secondary goals. The group-based cognitive behavioural therapy, consisting of eleven sessions over two years, targeted QoL and self-management of physical activity and eating [26]. Scheduled physical activity in the residential periods consisted of brisk walking, swimming, strength training, ball games, and aerobics and amounted to nine to eleven hours weekly divided into bouts of 20-60 minutes. Each patient developed a plan for physical activity, modified to his or her preferences, limitations, and home situation. The diet followed the Nordic Nutrition Recommendations [27] and consisted of three high-fibre, low-fat, and energy-reduced meals and two to three snacks. The patients were advised to follow a similar diet at home. In the home periods, patients kept physical activity diaries which they sent to RCHRC on a monthly basis. There was no other follow-up between the residential periods.

\subsection{Quality of Life Measures}

2.2.1. SF-36, Version 1.2. This is a 36-item measure of general health-related QoL. PCS and MCS are computed from the eight SF-36 subscales. PCS ranges from 15.4 to 62.1 and MCS from 10.1 to 64.0 (with higher scores representing better QoL) $[28,29]$. The PCS and MCS have been standardised to a population normal distribution, with a mean of 50 and a standard deviation (SD) of 10 . SF-36 has been widely applied in obesity research $[1,30]$, discriminates between subgroups of severely obese adults [1], and is sensitive to change during lifestyle interventions $[4,5]$.

2.2.2. OP Scale, Version 1.2. This is an eight-item measure of obesity-specific QoL including questions about restaurant visits, holidaying, participation in community activities, swimming in public places, trying on and buying clothes, and intimate/sexual situations. The calibrated score ranges from 0 to 100 ( $<40$ mild, $\geq 40$ to $<60$ moderate, $\geq 60$ to $<80$ severe, and $\geq 80$ extreme problems) [2]. OP is reliable and valid in severely obese adults $[2,31]$. In the present study, the internal consistency at baseline was excellent with a Cronbach alpha coefficient of 0.91 . 
2.2.3. Life Satisfaction. Life satisfaction was assessed using a global question on current satisfaction with life with seven response alternatives from "very satisfied" to "very dissatisfied." One-item measures on life satisfaction have demonstrated reliability [32] and validity in health research $[33,34]$.

2.3. Physical Activity. To assess physical activity, we used the ActiGraph GTI M accelerometer (ActiGraph, Pensacola, FL, USA), which is an electronic movement sensor. The accelerometer registers vertical acceleration and converts it into the unit "counts" which increase with the magnitude of the work rate for walking. The participants were instructed to wear the accelerometer over the right hip for seven consecutive days while awake, except during water activities. The $T 0, T 2$, and $T 3$ assessments took place during home periods, while the $T 1$ assessment was carried out at the end of the first residential period. The ActiGraph software ActiLife v. 5.3 was used for the data analysis. The criterion for a valid measure was wear-time of $\geq$ ten hours per day for $\geq$ four days. Non-wear-time was defined as periods of $\geq 60$ consecutive minutes without counts, allowing for up to two minutes of counts within these 60 minutes $[35,36]$. The overall physical activity, given as counts per minute, was calculated as total counts divided by total valid wear-time. The accelerometer has shown validity in severely obese individuals [37] and accelerometer assessed physical activity offers more accuracy than self-reported data [38].

2.4. Sociodemographic Information and Anthropometry. Sociodemographic information was self-reported on questionnaires. Health professionals collected the anthropometric data. Height was measured in the standing position without shoes using a stadiometer and reported to the nearest $0.5 \mathrm{~cm}$. Fat mass and weight were measured on a bioelectrical impedance analysis device (BC 420S MA, Tanita Corp., Tokyo, Japan) in the morning, in a fasting state, in light clothes, and after voiding. Weight was reported to the nearest $0.1 \mathrm{~kg}$. Waist circumference was measured twice at exhalation at the level of the umbilicus and reported as the mean of the two measurements.

2.5. Ethics. We obtained written, informed consent from all participants prior to the study in accordance with the Helsinki Declaration. Ethical approval was given by the Regional Committee for Medical and Health Research Ethics for South-East Norway (registration number 2010/159).

2.6. Statistical Analysis. The scores on life satisfaction were reversed before analyses so that higher scores indicated better satisfaction with life. BMI was calculated as weight in kilograms divided by height in meters squared. Subject characteristics are presented as means and SD for continuous variables and percentages for categorical data. Observed values for QoL measures are presented as means and SD. The effect size (ES) for differences between PCS and MSC population norms [39] and the study population scores at the four time points were calculated by subtracting the norms from the mean score of the participants divided by the SD of the latter. We performed an attrition analysis using the
TABLE 1: Characteristics of the adults with severe obesity at baseline, $N=49$.

\begin{tabular}{lc}
\hline Age, mean (SD) & $43.6(9.4)$ \\
Gender, $n$ (\%) & $37(75.5)$ \\
$\quad$ Women & \\
Sociodemographic status, $n$ (\%) & $31(63.3)$ \\
$\quad$ Married/cohabiting & $27(55.1)$ \\
Having children & $22(44.9)$ \\
College/university education & $41(83.7)$ \\
Employed & \\
Anthropometrics, mean (SD) & $42.1(6.0)$ \\
Body mass index, kg/m ${ }^{2}$ & $123.9(18.6)$ \\
Weight, kg & $128.3(13.0)$ \\
Waist circumference, cm & $58.2(11.7)$ \\
Fat mass, $\%$ & \\
\hline
\end{tabular}

Standard deviation: SD.

chi-squared test for difference in gender and the independent samples $t$-test for differences in other variables.

A linear mixed model based on restricted maximum likelihood estimation with random intercept for subjects was used in all analyses for change $(\Delta)$ over time [40], using least significant difference from baseline. The associations between the independent and the dependent variables were analysed using linear regression, applying delta scores between each time point $\left(\Delta y_{1}=y_{1}-y_{0} ; \Delta x_{1}=x_{1}-x_{0} ; \Delta y_{2}=y_{2}-y_{1}\right.$, etc. $)$ [40], giving a total of $n=73$ (PCS and MCS), 72 (OP), and 71 (life satisfaction) observations. For physical activity, PCS, MCS, OP, life satisfaction, and BMI, the differences between $T 0$ and $T 1(\Delta 1), T 1$ and $T 2(\Delta 2)$, and $T 2$ and $T 3(\Delta 3)$ were used. Gender, age, and change in BMI served as covariates in the adjusted regression analyses. A 1000-repetition bootstrap analysis was used to calculate $95 \%$ confidence intervals (CI) of the regression coefficients.

The changes from baseline to each of the time points in QoL measures, physical activity, and BMI, obtained from the linear mixed model, are presented as means with $95 \%$ CI. The ES for changes in the dependent variables were calculated by subtracting the mean $T 1, T 2$, and $T 3$ estimates from the mean $T 0$ estimate, divided by the SD of T0. Weight loss was calculated as percent change from baseline. A secondary analysis was performed using a baseline-observation-carried-forward approach for missing values.

Effect sizes were judged against the standard criteria proposed by Cohen: trivial $(<0.2)$, small $(0.2$ to $<0.5)$, moderate $(0.5$ to $<0.8)$, and large $(\geq 0.8)$ [41].

Calculation of sample size and power was done using the GPower version 3.1. Statistical analyses were conducted using SPSS for Windows, version 20.0 (SPSS Inc., Chicago, USA). A two-sided $P$ value of $\leq 0.05$ indicated statistical significance.

\section{Results}

Forty-nine patients (37 women; $43.6 \pm 9.4$ years; BMI $42.1 \pm$ $6.0 \mathrm{~kg} / \mathrm{m}^{2}$ ) consented to participate in the study. Baseline sociodemographic and anthropometric characteristics are presented in Table 1. At year two, 16 women and six men 


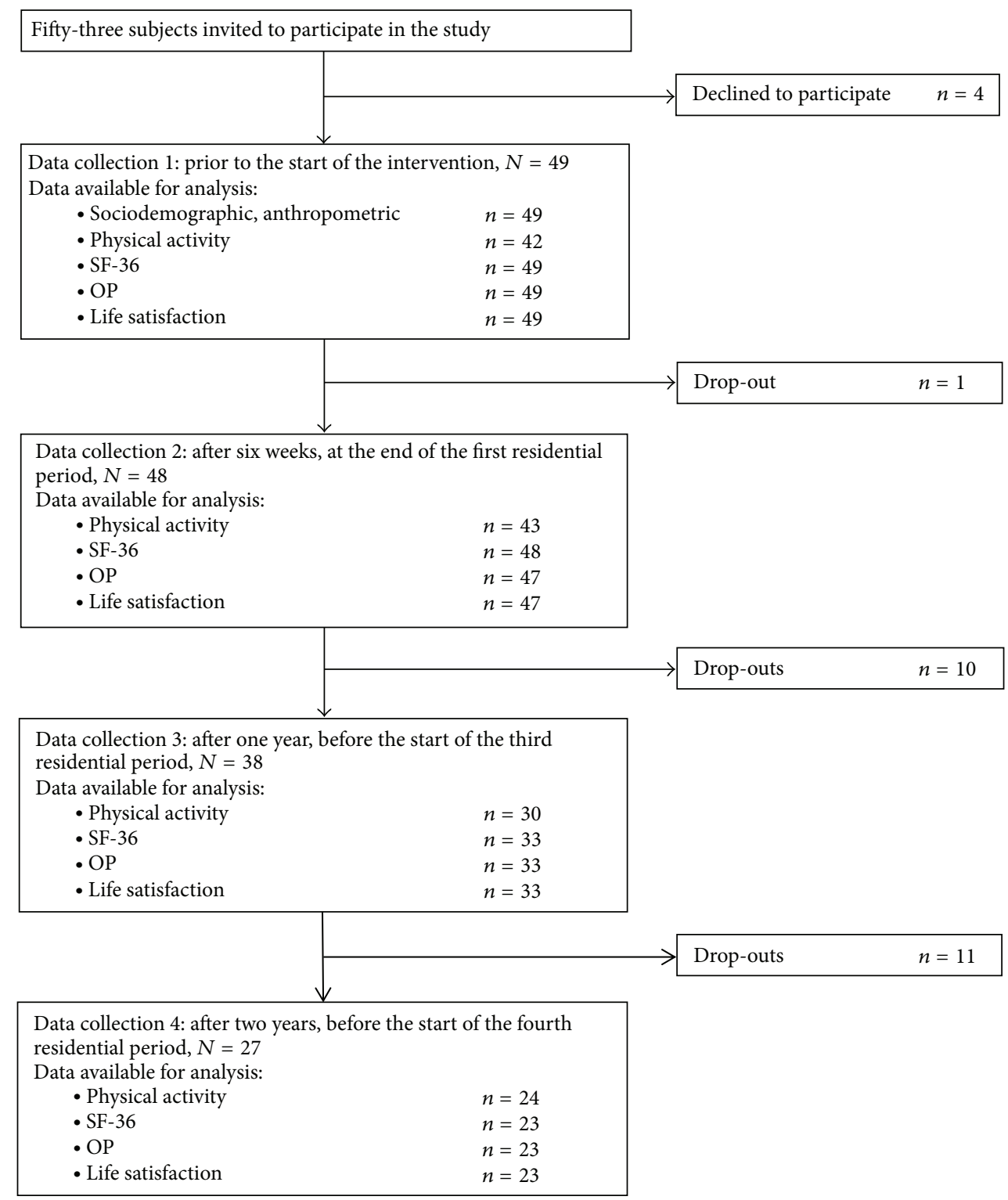

FIGURE 1: Flow chart for the prospective study of severely obese adults in a two-year lifestyle intervention, Medical Outcomes Study ShortForm 36 Health Survey: SF-36; Obesity-Related Problems (OP) scale.

(44.9\%) were lost to follow-up (Figure 1). Five withdrew from the study due to problems with the study protocol. The rest dropped out of the intervention itself, due to referral to obesity surgery, pregnancy, reaching personal weight goals, health problems, inability to attend the residential stays, or unknown reasons. The number of participants at each time point is noted in Table 2. The noncompleters did not differ from the completers with regard to gender, age, BMI, physical activity, QoL measures, or changes from $T 0$ to $T 1$ in BMI, physical activity, or QoL measures.

Table 2 documents changes in the QoL measures and the related ES. Over the first six weeks, all scores improved significantly. After two years, MCS and life satisfaction had returned to baseline levels, whereas the improvement in PCS was partly maintained. OP showed a different pattern with continuous improvements. The ES for within-group change was small for OP, moderate for PCS and MCS, and large for life satisfaction after six weeks. At year one, the ES was small for OP and moderate for PCS. Finally, at year two, the ES were small for PCS and moderate for OP [41]. Physical activity increased significantly during the first residential stay and was partly maintained at year one. At year two, it had returned to baseline level. Weight loss peaked at year one with $6.4 \%$ (Table 3).

Scores on PCS, MCS, OP, and life satisfaction are presented in Table 4 including ES for differences between the study population and the Norwegian SF-36 population norm [39]. For PCS, the difference was small at baseline and trivial at year two [41]. For MCS, it was trivial both at baseline and after two years. At baseline, the participants reported 
TABLE 2: Mixed-effect model estimates: mean changes (95\% CI) in quality of life outcomes during the two-year lifestyle intervention for severely obese adults.

\begin{tabular}{|c|c|c|c|}
\hline Measure & $T 1$ : change from $T 0$ & $T 2$ : change from $T 0$ & T3: change from $T 0$ \\
\hline SF-36 physical component summary ${ }^{\mathrm{a}}$ & $5.7(7.4,4.0) P<.001$ & $4.4(6.3,2.5) P<.001$ & $3.3(5.5,1.1) P=.004$ \\
\hline Effect size & 0.61 & 0.54 & 0.48 \\
\hline SF-36 mental component summary ${ }^{\mathrm{b}}$ & $5.9(8.6,3.1) P<.001$ & $-0.4(-2.7,3.5) P=.794$ & $-1.8(-1.8,5.3) P=.327$ \\
\hline Effect size & 0.55 & 0.02 & -0.06 \\
\hline Obesity-related problems scale ${ }^{c}$ & $-8.4(-2.9,-13.9) P=.003$ & $-11.2(-5.0,-17.4) P=.001$ & $-13.3(-6.2,-20.4) P<.001$ \\
\hline Effect size & 0.30 & 0.49 & 0.57 \\
\hline Life satisfaction ${ }^{\mathrm{d}}$ & $0.99(1.30,0.67) P<.001$ & $0.47(0.82,0.12) P=.009$ & $0.20(0.60,0.20) P=.324$ \\
\hline Effect size & 1.00 & 0.55 & 0.22 \\
\hline
\end{tabular}

T0: before the intervention $(n=49)$; T1: after six weeks $(n=48)$; T2: year one $(n=38)$; T3: year two $(n=27)$.

${ }^{a}$ Scale 15.4-62.1: higher scores represent better quality of life, Medical Outcomes Study Short-Form 36 Health Survey.

${ }^{\mathrm{b}}$ Scale 10.1-64.0: higher scores represent better quality of life, Medical Outcomes Study Short-Form 36 Health Survey.

${ }^{c}$ Scale 0-100: higher scores represent more obesity-related problems.

${ }^{\mathrm{d}}$ Scale 1-7: higher scores represent better life satisfaction.

Significant $P$ values ( $\leq .05)$ in bold.

Effect sizes for the within-group changes were calculated by subtracting the mean estimates of follow-ups from the mean estimates at baseline divided by the SD of the latter. They were judged against the standard criteria proposed by Cohen: trivial $(<0.2)$, small $(0.2$ to $<0.5)$, moderate $(0.5$ to $<0.8)$, and large $(\geq 0.8)$ [41].

Confidence interval: $\mathrm{CI}$.

TABLE 3: Mixed-effect model estimates: physical activity and BMI during the two-year lifestyle intervention for severely obese adults.

\begin{tabular}{|c|c|c|c|c|c|c|c|}
\hline & $\begin{array}{c}\text { T0 } \\
\text { Mean }(95 \% \text { CI })\end{array}$ & $\begin{array}{c}T 1 \\
\text { Mean }(95 \% \mathrm{CI}) \\
\end{array}$ & $P^{*}$ & $\begin{array}{c}T 2 \\
\text { Mean }(95 \% \mathrm{CI}) \\
\end{array}$ & $P^{*}$ & $\begin{array}{c}\text { T3 } \\
\text { Mean }(95 \% \text { CI }) \\
\end{array}$ & $P^{*}$ \\
\hline Accelerometer assessed physical activity, $\mathrm{CPM}^{\dagger}$ & $\begin{array}{c}n=42 \\
276(241,311)\end{array}$ & $\begin{array}{c}n=43 \\
452(417,486)\end{array}$ & $<.001$ & $\begin{array}{c}n=30 \\
327(286,368)\end{array}$ & .036 & $\begin{array}{c}n=24 \\
290(244,335)\end{array}$ & .606 \\
\hline BMI, kg/m² & $\begin{array}{c}n=49 \\
42.1(40.3,43.8)\end{array}$ & $\begin{array}{c}n=48 \\
40.1(38.4,41.8)\end{array}$ & $<.001$ & $\begin{array}{c}n=38 \\
39.4(37.6,41.1)\end{array}$ & $<.001$ & $\begin{array}{c}n=27 \\
40.7(38.9,42.5)\end{array}$ & .001 \\
\hline Weight loss from $T 0$, per cent & & 4.8 & & 6.4 & & 3.3 & \\
\hline
\end{tabular}

$T 0$ : before the intervention; $T 1$ : after six weeks; $T 2$ : year one; $T 3$ : year two.

Significant $P$ values $(\leq .05)$ in bold.

${ }^{*} P$ values for change from $T 0$.

Body mass index: BMI; confidence interval: CI; counts per minute: CPM.

${ }^{\dagger}$ Mean physical activity of American obese adults: 288 CPM [58]. Mean physical activity of Norwegian obese women: 276 CPM, and men: 290 CPM [59]. Mean physical activity of American normal weight adults: 344 CPM [58]. Mean physical activity of Norwegian normal weight women: 352 CPM, and men: 368 CPM [59].

TABLE 4: Mean and standard deviation of quality of life outcomes during the two-year lifestyle intervention for severely obese adults.

\begin{tabular}{|c|c|c|c|c|c|}
\hline & T0 & $T 1$ & $T 2$ & T3 & Population norm \\
\hline SF-36 ${ }^{\mathrm{a}}$ & $n=49$ & $n=47$ & $n=33$ & $n=23$ & \multirow{3}{*}{49.0} \\
\hline Physical component summary & $45.3(9.6)$ & $51.2(7.3)$ & $50.5(7.8)$ & $49.9(7.6)$ & \\
\hline Effect size & -0.39 & 0.30 & 0.19 & 0.12 & \\
\hline Mental component summary & $48.4(10.2)$ & $54.0(7.7)$ & $48.6(12.8)$ & $47.8(10.7)$ & \multirow{2}{*}{49.0} \\
\hline Effect size & -0.06 & 0.65 & -0.03 & -0.11 & \\
\hline Obesity-related problems $\mathrm{s}^{\mathrm{b}}$ & $n=49$ & $n=46$ & $n=33$ & $n=23$ & \multirow{2}{*}{$\mathrm{n} / \mathrm{a}$} \\
\hline & $44.6(26.3)$ & $36.7(25.0)$ & $31.8(28.0)$ & $29.7(24.4)$ & \\
\hline Life satisfaction ${ }^{c}$ & $n=49$ & $n=46$ & $n=33$ & $n=23$ & \multirow{2}{*}{$\mathrm{n} / \mathrm{a}$} \\
\hline & $4.6(0.9)$ & $5.5(1.0)$ & $5.1(0.9)$ & $4.8(0.9)$ & \\
\hline
\end{tabular}

T0: before the intervention; T1: after six weeks; T2: year one; T3: year two.

${ }^{a}$ Scale 0-100: higher scores represent better quality of life, Medical Outcomes Study Short-Form 36 Health Survey (SF 36). The SF-36 data for the norm population $(n=2,323)$ are adjusted for gender and age [39] and presented as means. Effect sizes for differences between the study participants and the norm population were calculated by subtracting the mean score of the population norm from the mean score of the study participants divided by the SD of the latter. They were judged against the standard criteria proposed by Cohen: trivial $(<0.2)$, small $(0.2$ to $<0.5)$, moderate $(0.5$ to $<0.8)$, and large $(\geq 0.8)$ [41].

${ }^{\mathrm{b}}$ Scale 0-100: higher scores represent more obesity-related problems, Obesity-Related Problems scale.

${ }^{c}$ Scale 1-7: higher scores represent better life satisfaction. 


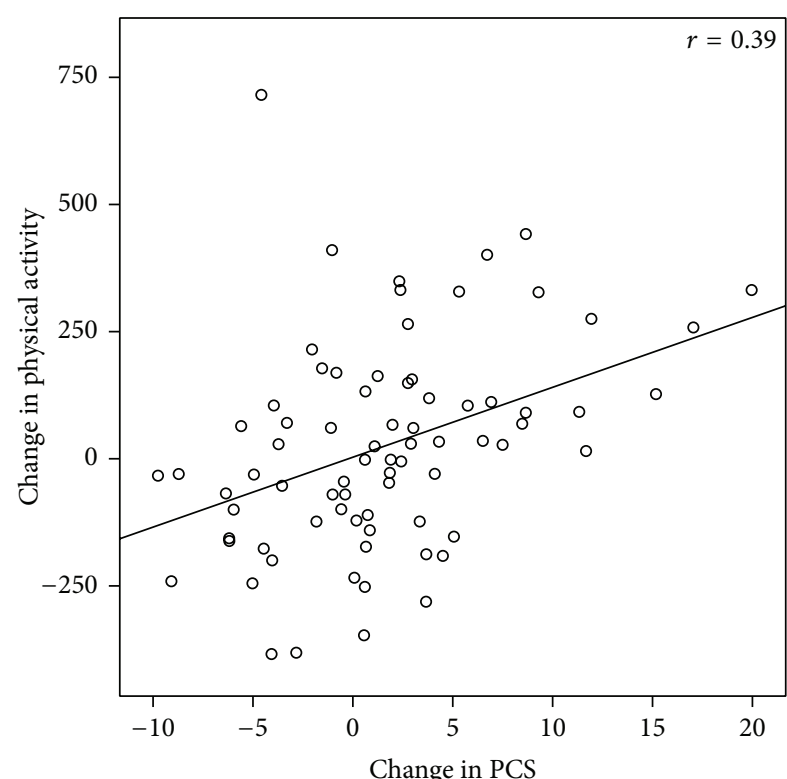

(a)

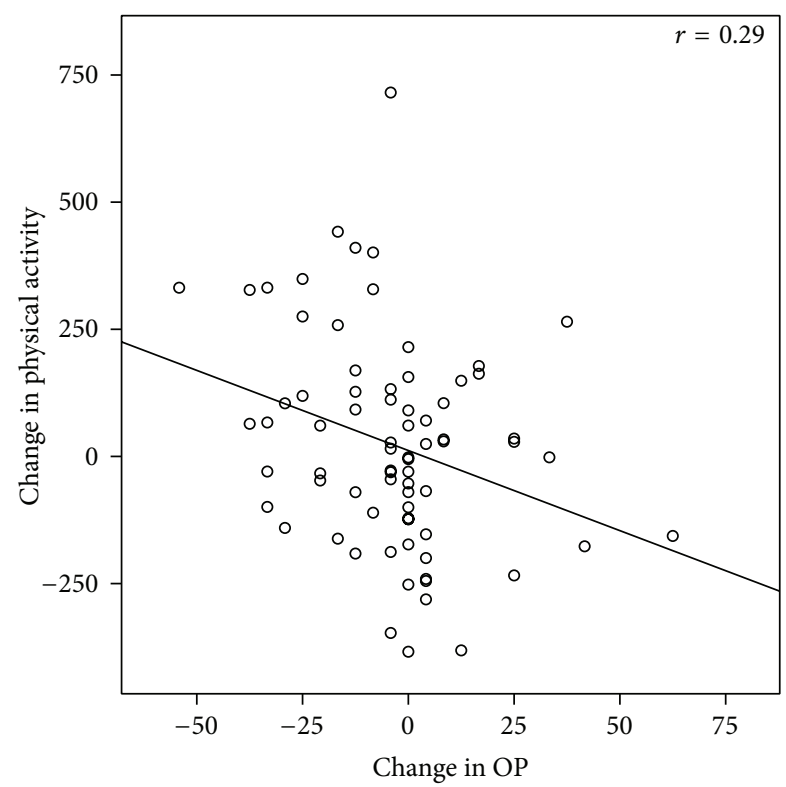

(c)

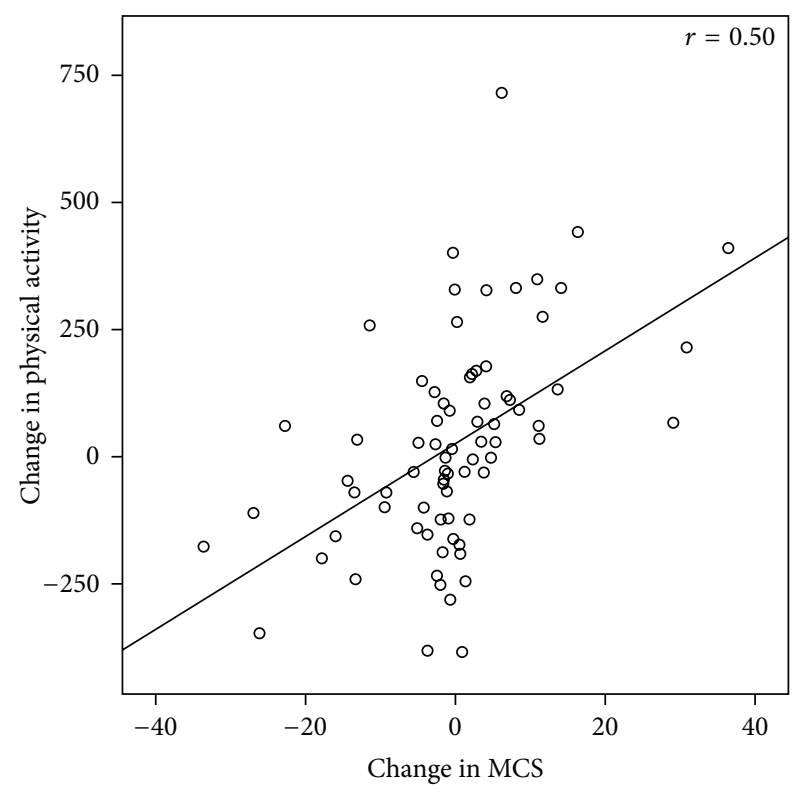

(b)

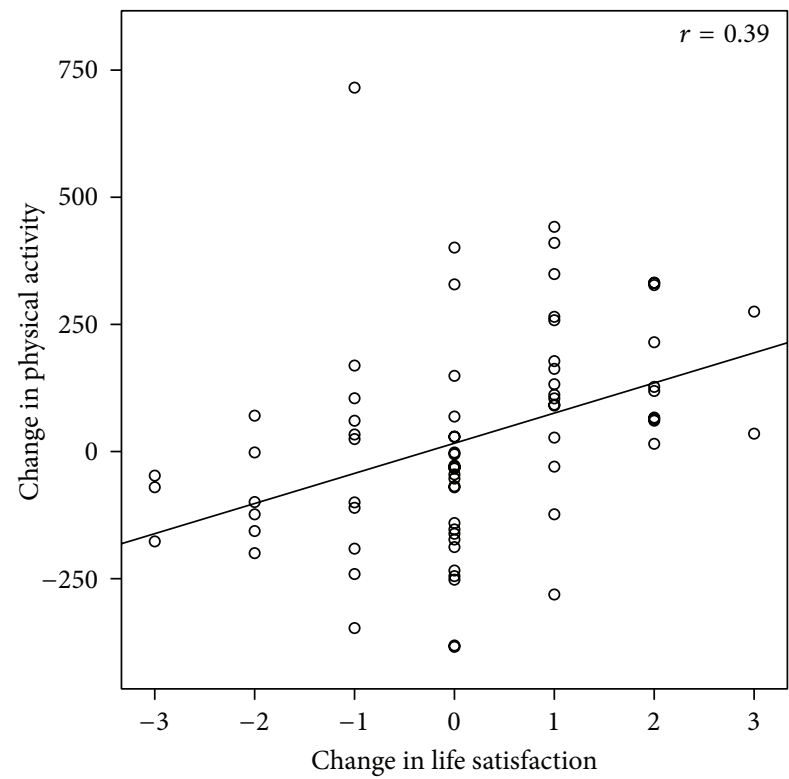

(d)

FIGURE 2: (a)-(d) Correlations between change in accelerometer assessing physical activity (counts per minute) and change in (a) PCS, (b) MCS, (c) OP, and (d) life satisfaction during the two-year lifestyle intervention for severely obese adults. PCS (physical component summary) score of the Medical Outcomes Study Short-Form 36 Health Survey (SF-36). Scale: 15.4-62.1. Higher scores represent better health-related quality of life. MCS (mental component summary) score of the Medical Outcomes Study Short-Form 36 Health Survey (SF-36). Scale: 10.164.0. Higher scores represent better health-related quality of life. OP: Obesity-Related Problems scale. Scale: 0-100. Higher scores represent more obesity-related problems. Life satisfaction. Scale: 1-7. Higher scores represent better satisfaction with life.

moderate obesity-related problems. Thereafter, the scores on OP reduced to mild problems [2].

Figure 2 illustrates that correlations between change in physical activity and change in QoL measures were strongest for MCS and weakest for OP. This is also demonstrated in Table 5 which presents the results of the regression analyses. In the adjusted analyses, changes in PCS, MCS, OP, and life satisfaction were significantly associated with change in physical activity. The explained variance was moderate for PCS and MCS and small for OP and life satisfaction [41]. Change in BMI was correlated with change in PCS, MCS, and life satisfaction in the unadjusted analyses; however, this association was not statistically significant in the full models. Replacement of change in BMI with change in waist circumference or fat mass did not alter any results (data not shown). We tested for the interaction between physical 


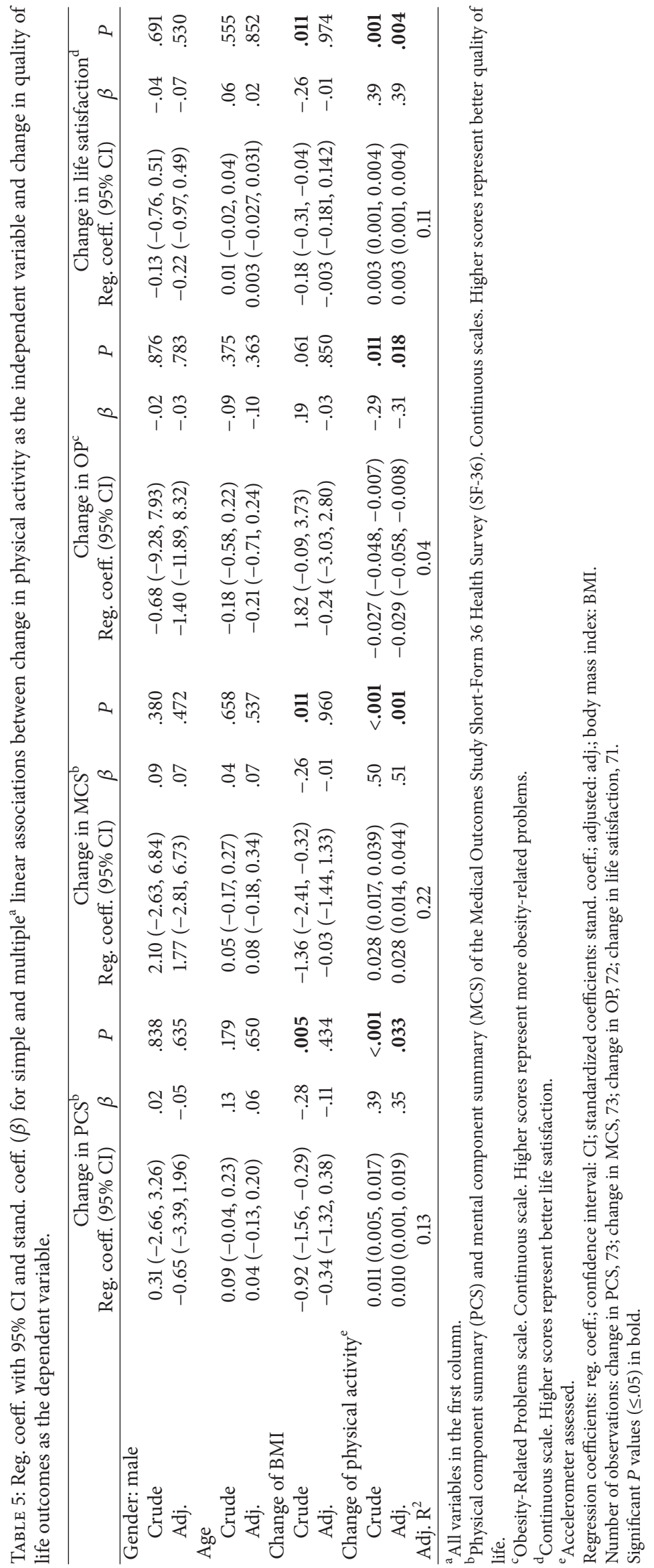


activity and gender in all four models and found that the women had a stronger association between change in physical activity and change in PCS than the men $(P=0.012)$.

This study has 71 to 73 observations for the main outcomes (Table 5). Given 71 observations, a power of 0.80 , and significance level of 0.05 , the study should have power to detect a standardized coefficient of 0.32 (medium effect size).

\section{Discussion}

Results from this study suggest that change in physical activity is independently associated with change in physical, mental, and obesity-specific QoL and life satisfaction in severely obese adults participating in a lifestyle intervention. In correspondence with the present study, an independent dose-response relationship between exercise and physical and mental SF-36 subscales was reported from a randomized, controlled pre-post study on a six-month intervention for overweight to obese, menopausal, and hypertensive women [42]. A cross-sectional study on overweight to obese subjects with type 2 diabetes also reported associations between selfreported physical activity and MCS, but not PCS, independent of BMI [43]. By contrast, Ross et al. [44] reported that physical fitness, which may be a proxy for physical activity, did not mediate the association between weight reduction and improvements in SF-36 subscales in obese women enrolled in a six-month intervention. However, the six-minute walk test, which was utilized to assess fitness in the study by Ross et al. [44], may lack accuracy in pre-post design in obesity research [21]. Bond et al. [19] used self-reported data on physical activity from two time points and found associations with PCS, but not MCS, in obesity surgeryseekers, although these results did not control for BMI. With respect to obesity-specific QoL, Kaukua et al. [6] described improvements in OP alongside fluctuations in self-reported physical activity during a lifestyle intervention but did not examine correlations. Regarding life satisfaction, our crosssectional baseline study from the Haugland Obesity Study found an independent association with physical activity [20]. Population data revealed a positive relationship with selfreported physical activity, though this relationship did not control for BMI [10]. A comparison of lifestyle interventions found no difference in life satisfaction between intervention and control groups during a one-year follow-up [35]. However, physical activity was not included in the analyses. So some studies do not support our finding of associations between change in physical activity and change in all QoL outcomes. One explanation may be that the intervention of the present study clearly differed from other lifestyle interventions in that the overall goal was improvement of QoL. Other interventions have weight management $[2,7]$ or behaviour change related to physical activity and diet (which should lead to weight loss) [4-6] as primary goals. The inconsistencies across studies may also relate to the crosssectional design of several of them $[10,20,43]$, problems with the reliability of self-reported physical activity [36], the variety of weight classes included in the studies, and other heterogeneities of participants, context, or research designs.
Interestingly, although the unadjusted analyses revealed correlations between changes in BMI and PCS, MCS, and life satisfaction, weight loss did not moderate the associations between the independent and the dependent variables in the adjusted analyses. Associations between weight loss and improvements in PCS have been found in several studies on patients undergoing lifestyle interventions $[4,5,37]$ but not in the study by Blissmer et al. [7]. Findings on the relationship between weight loss and MCS are also inconsistent. Neither Danielsen et al. [4] nor Pazzagli et al. [37] found this association in lifestyle interventions. Neither did Kolotkin et al. [38] after obesity surgery, whereas Karlsen et al. [5] did in a pooled sample of obesity surgery patients and lifestyle intervention completers. Our finding on OP, as the only variable which had a nonsignificant correlation with change in BMI in the unadjusted analyses, is contradictive to the SOS study which demonstrated short- and long-term decreases in obesity-related problems associated with weight loss $[2,8]$. Other obesity-specific measures have also shown associations with weight loss [38]. More research is needed to fully understand the relationship between weight loss and QoL. Noticeably, with regard to the purpose of the present study, none of the above-mentioned studies included physical activity as a variable.

The positive boost in all QoL measures over the first residential stay is noteworthy. PCS and MCS even increased above population norms [39]. Similar improvements on PCS and MCS have been found by Danielsen et al. [4] after an initial, in-patient period and Kaukua et al. [6] at the end of a four-month outpatient programme using the RAND36 questionnaire, equivalent to the SF-36 [45], and the OP. However, despite the statistically significant effect of physical activity on QoL measures in our analyses, many of the improvements in the dependent variables were left unexplained. Thus, several other aspects may have played a role, such as experience of peer support $[46,47]$, reduction of anxiety [4], improved eating pattern [48], and improved self-regulation and self-efficacy [49] which have been demonstrated by others after intensive intervention phases, as well as our explicit intervention focus on improvement of QoL. Regarding the patterns of longer-term changes, there are variations across studies. Our finding that MCS had returned to baseline at year two while moderate ES for the change in PCS was maintained is opposite to two-year changes reported by Blissmer el al. [7] on overweight to obese subjects. The ES for one-year changes reported for lifestyle intervention completers by Karlsen et al. [5] is similar to the ES of the present study for PCS (=0.47), but higher $(=0.32)$ for MCS. Yet the baseline scores on PCS (mean $=39, \mathrm{SD}=10$ ) and MCS (mean $=42, \mathrm{SD}=11$ ) were lower compared to our study which may indicate a greater potential for long-term improvements. So, although lifestyle treatment-seekers generally report better QoL than obesity surgery-seekers and worse than nontreatment-seekers $[1,3]$, variations across study populations in lifestyle interventions may contribute to disparities in research outcomes. Moreover, it may be unrealistic to expect the initial peaks in PCS, MCS, and life satisfaction to last in the long run. In fact, that would imply better PCS and MCS scores than in the general population [39]. For future studies, 
examining how favourable outcomes of intensive phases of lifestyle interventions can be maintained, at least partly, over time will be worthwhile. And, in that regard, our finding of continued improvement in the OP is of interest. An intervention that can help participants experience less obesity-related problems, despite modest weight loss, may be seen as positive.

Change of health-related behaviours is challenging [13] and the interaction of time is an aspect that deserves consideration. Often, lifestyle interventions are reported to be shorter than the present, for example, four months [6], six months [7], or one year $[4,5]$. The SOS study has published data on long-term follow-up, but the length of the included conventional treatment is not standardised or described $[2,8]$. The Look AHEAD study, though including patients of all weight classes from overweight to severely obese, does provide detailed information about lifestyle intervention for adults with type 2 diabetes and is unique with regard to the large number of participants $(N=5,145)$, the randomisation, and the length of the intervention, that is, eight years [17]. Clearly, more studies are needed to develop a better understanding of long-term effects of lifestyle interventions on QoL outcomes.

4.1. Methodological Considerations. The present study has several strengths. First, the use of accelerometers to collect data on physical activity increased reliability over selfreporting [36]. Second, as recommended, both general and condition-specific QoL instruments were used [2]. Third, data was collected at four time points and used to reveal associations of patterns of change. Since change of healthrelated behaviour is complex, increase and maintenance of physical activity are challenging, and subjective constructs like QoL are not straightforward, these findings on patterns of change contribute uniquely to the body of knowledge about lifestyle interventions for severely obese adults.

A limitation of this study was that the number of participants lost to follow-up challenged the statistical power. High attrition is not unusual in research on lifestyle interventions $[6,7]$ but differences between completers and noncompleters vary across studies from none $[5,6]$ to one $[4,7]$ or some [50]. In the present study, the attrition analyses revealed no statistical difference in key variables between the dropouts and the completers. The secondary analysis confirmed the statistical level of change in the QoL measures, counts per minute, and BMI (data not shown). To deal with the challenge of statistical power, all valid data were included in the linear mixed model. Regarding the sample size, the study was powered to detect medium sized effect sizes as found in the regression analysis of this study. Inclusion of a control group amongst the referred patients was not possible due to their right to treatment [24]. This study examined associations and, therefore, causal relationships could not be inferred. And the study did not control for change of diet, a possible confounder in the associations we examined [51, 52]. However, physical activity has been found to contribute more to QoL than dieting [53]. In addition, we controlled for change in BMI which may be a proxy for diet, because generally diet modifications produce more weight loss than physical activity $[54,55]$. Lastly, the patients were a selfselected, treatment-seeking group although public funding of the intervention gave equal access to all and, therefore, diminished the risk of socioeconomic bias [56].

\section{Conclusions}

It has been proposed that lifestyle interventions for obese individuals should focus less on weight loss as the primary outcome and pay more attention to independent benefits of physical activity such as reduction of obesity-related health hazards [57] and improvements of QoL [42]. The present study contributes uniquely to the literature on severe obesity, physical activity, and self-reported outcomes and indicates that improved QoL may be a valid result of increased physical activity in multicomponent lifestyle interventions. These findings should be further tested in various settings, in larger samples, and with control groups.

\section{Conflict of Interests}

The authors declare that there is no conflict of interests regarding the publication of this paper.

\section{Acknowledgments}

The authors thank Sogn and Fjordane University College for funding the study, the staff at RCHR for their help with the data collection, and the participants for their invaluable contributions.

\section{References}

[1] A. M. A. van Nunen, E. J. M. Wouters, A. J. J. M. Vingerhoets, J. J. Hox, and R. Geenen, "The health-related quality of life of obese persons seeking or not seeking surgical or non-surgical treatment: a meta-analysis," Obesity Surgery, vol. 17, no. 10, pp. 1357-1366, 2007.

[2] J. Karlsson, C. Taft, L. Sjöström, J. S. Torgerson, and M. Sullivan, "Psychosocial functioning in the obese before and after weight reduction: construct validity and responsiveness of the Obesityrelated Problems scale," International Journal of Obesity, vol. 27, no. 5, pp. 617-630, 2003.

[3] R. L. Kolotkin, R. D. Crosby, and G. R. Williams, "Health-related quality of life varies among obese subgroups," Obesity Research, vol. 10, no. 8, pp. 748-756, 2002.

[4] K. K. Danielsen, J. Sundgot-Borgen, S. Maehlum, and M. Svendsen, "Beyond weight reduction: improvements in quality of life after an intensive lifestyle intervention in subjects with severe obesity," Annals of Medicine, vol. 46, no. 5, pp. 273-282, 2014.

[5] T. I. Karlsen, R. S. Lund, J. Røislien et al., "Health related quality of life after gastric bypass or intensive lifestyle intervention: a controlled clinical study," Health and Quality of Life Outcomes, vol. 11, article 17, 2013.

[6] J. Kaukua, T. Pekkarinen, T. Sane, and P. Mustajoki, "Healthrelated quality of life in obese outpatients losing weight with very-low-energy diet and behaviour modification-a 2-y follow-up study," International Journal of Obesity and Related Metabolic Disorders, vol. 27, no. 10, pp. 1233-1241, 2003.

[7] B. Blissmer, D. Riebe, G. Dye, L. Ruggiero, G. Greene, and M. Caldwell, "Health-related quality of life following a clinical 
weight loss intervention among overweight and obese adults: intervention and 24 month follow-up effects," Health and Quality of Life Outcomes, vol. 4, article 43, 2006.

[8] J. Karlsson, C. Taft, A. Rydén, L. Sjöström, and M. Sullivan, "Ten-year trends in health-related quality of life after surgical and conventional treatment for severe obesity: the SOS intervention study," International Journal of Obesity, vol. 31, no. 8, pp. 1248-1261, 2007.

[9] I. McDowell, "Measures of self-perceived well-being," Journal of Psychosomatic Research, vol. 69, no. 1, pp. 69-79, 2010.

[10] T. W. Strine, D. P. Chapman, L. S. Balluz, D. G. Moriarty, and A. H. Mokdad, "The associations between life satisfaction and health-related quality of life, chronic illness, and health behaviors among U.S. community-dwelling adults," Journal of Community Health, vol. 33, no. 1, pp. 40-50, 2008.

[11] T. Wadsworth and P. M. Pendergast, "Obesity (sometimes) matters: the importance of context in the relationship between obesity and life satisfaction," Journal of Health and Social Behavior, vol. 55, no. 2, pp. 196-214, 2014.

[12] P. Schnohr, T. S. Kristensen, E. Prescott, and H. Scharling, "Stress and life dissatisfaction are inversely associated with jogging and other types of physical activity in leisure timethe Copenhagen City Heart Study," Scandinavian Journal of Medicine and Science in Sports, vol. 15, no. 2, pp. 107-112, 2005.

[13] S. F. L. Kirk, T. L. Penney, T.-L. F. McHugh, and A. M. Sharma, "Effective weight management practice: a review of the lifestyle intervention evidence," International Journal of Obesity, vol. 36, no. 2, pp. 178-185, 2012.

[14] C. T. Miller, S. F. Fraser, I. Levinger et al., "The effects of exercise training in addition to energy restriction on functional capacities and body composition in obese adults during weight loss: a systematic review," PLoS ONE, vol. 8, no. 11, Article ID e81692, 2013.

[15] J. M. R. Gill and D. Malkova, "Physical activity, fitness and cardiovascular disease risk in adults: interactions with insulin resistance and obesity," Clinical Science, vol. 110, no. 4, pp. 409425, 2006.

[16] M. Fogelholm, "Physical activity, fitness and fatness: Relations to mortality, morbidity and disease risk factors. A systematic review," Obesity Reviews, vol. 11, no. 3, pp. 202-221, 2010.

[17] The Look AHEAD Research Group, "Eight-year weight losses with an intensive lifestyle intervention: the look AHEAD study," Obesity, vol. 22, no. 1, pp. 5-13, 2014.

[18] A. Lerdal, R. Andenæs, E. Bjørnsborg et al., "Personal factors associated with health-related quality of life in persons with morbid obesity on treatment waiting lists in Norway," Quality of Life Research, vol. 20, no. 8, pp. 1187-1196, 2011.

[19] D. S. Bond, R. K. Evans, E. DeMaria et al., "Physical activity and quality of life improvements before obesity surgery," American Journal of Health Behavior, vol. 30, no. 4, pp. 422-434, 2006.

[20] R. Jepsen, E. Aadland, J. R. Andersen, and G. K. Natvig, "Associations between physical activity and quality of life outcomes in adults with severe obesity: a cross-sectional study prior to the beginning of a lifestyle intervention," Health and Quality of Life Outcomes, vol. 11, no. 1, article 187, 2013.

[21] E. Aadland, R. Jepsen, J. R. Andersen, and S. A. Anderssen, "Increased physical activity improves aerobic fitness, but not functional walking capacity, in severely obese subjects participating in a lifestyle intervention," Journal of Rehabilitation Medicine, vol. 45, no. 10, pp. 1071-1077, 2013.

[22] E. Aadland, R. Jepsen, J. R. Andersen, and S. A. Anderssen, "Differences in fat loss in response to physical activity among severely obese men and women," Journal of Rehabilitation Medicine, vol. 46, no. 4, pp. 363-369, 2014.

[23] E. Aadland, J. R. Andersen, S. A. Anderssen, and O. M. Kvalheim, "Impact of physical activity and diet on lipoprotein particle concentrations in severely obese women participating in a 1-year lifestyle intervention," Clinical Obesity, vol. 3, no. 6, pp. 202-213, 2013.

[24] Norwegian Directorate of Health, Sykelig overvekt: Prioriteringsveileder, Norwegian Directorate of Health, Oslo, Norway, 2009.

[25] R. Jepsen, E. Aadland, L. Robertson, M. Kristiansen, J. R. Andersen, and G. K. Natvig, "Factors and associations for physical activity in severely obese adults during a two-year lifestyle intervention," PeerJ, vol. 2, p. e505, 2014.

[26] E. K. Olander, H. Fletcher, S. Williams, L. Atkinson, A. Turner, and D. P. French, "What are the most effective techniques in changing obese individuals' physical activity self-efficacy and behaviour: a systematic review and meta-analysis," International Journal of Behavioral Nutrition and Physical Activity, vol. 10, article 29, 2013.

[27] W. Becker, N. Lyhne, A. N. Pedersen et al., "Nordic nutrition recommendations 2004-integrating nutrition and physical activity," Scandinavian Journal of Nutrition/Naringsforskning, vol. 48, no. 4, pp. 178-187, 2004.

[28] J. E. Ware, M. Kosinski, and B. Gandek, SF-36 Health Survey: Manual \& Interpretation Guide, QualityMetric, Lincoln, RI, USA, 2000.

[29] J. E. Ware and M. Kosinksi, SF-36 Physical and Mental Health Summary Scales: A Manual for Users of Version 1, QualityMetric Incorporated, Lincoln, RI, USA, 2001.

[30] Z. Ul-Haq, D. F. Mackay, E. Fenwick, and J. P. Pell, "Metaanalysis of the association between body mass index and healthrelated quality of life among adults, assessed by the SF-36," Obesity, vol. 21, no. 3, pp. E322-E327, 2013.

[31] A. Aasprang, J. R. Andersen, V. Våge, R. Kolotkin, and G. K. Natvig, "Reliability and validity of the Norwegian version of obesity-related problems scale," Obesity Facts, vol. 7, supplement 1, pp. 133-134, 2014.

[32] M. Zimmerman, C. J. Ruggero, I. Chelminski et al., "Developing brief scales for use in clinical practice: the reliability and validity of single-item self-report measures of depression symptom severity, psychosocial impairment due to depression, and quality of life," The Journal of Clinical Psychiatry, vol. 67, no. 10, pp. 1536-1541, 2006.

[33] S. Naess, J. Eriksen, K. Midthjell, and K. Tambs, "Subjective well-being before and after the onset of diabetes mellitus: results of the Nord-Trøndelag Health Study," Journal of Diabetes and Its Complications, vol. 19, no. 2, pp. 88-95, 2005.

[34] A.-S. Helvik, K. Engedal, S. Krokstad, and G. Selbæk, "A comparison of life satisfaction in elderly medical inpatients and the elderly in a population-based study: Nord-Trøndelag Health Study 3," Scandinavian Journal of Public Health, vol. 39, no. 4, pp. 337-344, 2011.

[35] S. Ash, M. Reeves, J. Bauer et al., "A randomised control trial comparing lifestyle groups, individual counselling and written information in the management of weight and health outcomes over 12 months," International Journal of Obesity, vol. 30, no. 10, pp. 1557-1564, 2006.

[36] S. A. Prince, K. B. Adamo, M. E. Hamel, J. Hardt, S. Connor Gorber, and M. Tremblay, "A comparison of direct versus self-report measures for assessing physical activity in adults: 
a systematic review," International Journal of Behavioral Nutrition and Physical Activity, vol. 5, article 56, 2008.

[37] C. Pazzagli, C. Mazzeschi, L. Laghezza, G. P. Reboldi, and P. De Feo, "Effects of a multidisciplinary lifestyle intervention for obesity on mental and physical components of quality of life: the mediatory role of depression," Psychological Reports, vol. 112, no. 1, pp. 33-46, 2013.

[38] R. L. Kolotkin, L. E. Davidson, R. D. Crosby, S. C. Hunt, and T. D. Adams, "Six-year changes in health-related quality of life in gastric bypass patients versus obese comparison groups," Surgery for Obesity and Related Diseases, vol. 8, no. 5, pp. 625633, 2012.

[39] J. H. Loge, S. Kaasa, M. J. Hjermstad, and T. K. Kvien, "Translation and performance of the Norwegian SF-36 Health Survey in patients with rheumatoid arthritis. I. Data quality, scaling assumptions, reliability, and construct validity," Journal of Clinical Epidemiology, vol. 51, no. 11, pp. 1069-1076, 1998.

[40] J. W. R. Twisk, Applied Longitudinal Data Analysis for Epidemiology: A Practical Guide, Cambridge University Press, Cambridge, UK, 2003.

[41] P. D. Ellis, The Essential Guide to Effect Sizes: Statistical Power, Meta-Analysis and the Interpretation of Research Results, Cambridge University Press, Cambridge, UK, 2011.

[42] C. K. Martin, T. S. Church, A. M. Thompson, C. P. Earnest, and S. N. Blair, "Exercise dose and quality of life," Archives of Internal Medicine, vol. 169, no. 3, pp. 269-278, 2009.

[43] K. Eckert, "Impact of physical activity and bodyweight on health-related quality of life in people with type 2 diabetes," Diabetes, Metabolic Syndrome and Obesity: Targets and Therapy, vol. 5, pp. 303-311, 2012.

[44] K. M. Ross, V. A. Milsom, K. A. Rickel et al., "The contributions of weight loss and increased physical fitness to improvements in health-related quality of life," Eating Behaviors, vol. 10, no. 2, pp. 84-88, 2009.

[45] R. D. Hays, C. D. Sherbourne, and R. M. Mazel, "The RAND 36-Item Health Survey 1.0," Health Economics, vol. 2, no. 3, pp. 217-227, 1993.

[46] S. Visram, A. Crosland, and H. Cording, "Triggers for weight gain and loss among participants in a primary care-based intervention," British Journal of Community Nursing, vol. 14, no. 11, pp. 495-501, 2009.

[47] U. Schulz, C. R. Pischke, G. Weidner et al., "Social support group attendance is related to blood pressure, health behaviours, and quality of life in the Multicenter Lifestyle Demonstration Project," Psychology, Health and Medicine, vol. 13, no. 4, pp. 423437, 2008.

[48] K. K. Danielsen, M. Svendsen, S. Mæhlum, and J. SundgotBorgen, "Changes in body composition, cardiovascular disease risk factors, and eating behavior after an intensive lifestyle intervention with high volume of physical activity in severely obese subjects: a prospective clinical controlled trial," Journal of Obesity, vol. 2013, Article ID 325464, 12 pages, 2013.

[49] J. J. Annesi, "Supported exercise improves controlled eating and weight through its effects on psychosocial factors: extending a systematic research program toward treatment development.", The Permanente journal, vol. 16, no. 1, pp. 7-18, 2012.

[50] P. J. Teixeira, S. B. Going, L. B. Houtkooper et al., "Pretreatment predictors of attrition and successful weight management in women," International Journal of Obesity and Related Metabolic Disorders, vol. 28, no. 9, pp. 1124-1133, 2004.

[51] C. S. Plaisted, P.-H. Lin, J. D. Ard, M. L. McClure, and L. P. Svetkey, "The effects of dietary patterns on quality of life: a substudy of the Dietary Approaches to Stop Hypertension trial," Journal of the American Dietetic Association, vol. 99, no. 8, pp. S84-S89, 1999.

[52] C. Ruano, P. Henriquez, M. A. Martínez-González, M. BesRastrollo, M. Ruiz-Canela, and A. Sánchez-Villegas, "Empirically derived dietary patterns and health-related quality of life in the SUN project," PLoS ONE, vol. 8, no. 5, Article ID e61490, 2013.

[53] M. K. Hassan, A. V. Joshi, S. S. Madhavan, and M. M. Amonkar, "Obesity and health-related quality of life: a cross-sectional analysis of the US population," International Journal of Obesity, vol. 27, no. 10, pp. 1227-1232, 2003.

[54] C. C. Curioni and P. M. Lourenço, "Long-term weight loss after diet and exercise: a systematic review," International Journal of Obesity, vol. 29, no. 10, pp. 1168-1174, 2005.

[55] T. Wu, X. Gao, M. Chen, and R. M. Van Dam, "Long-term effectiveness of diet-plus-exercise interventions vs. diet-only interventions for weight loss: a meta-analysis," Obesity Reviews, vol. 10, no. 3, pp. 313-323, 2009.

[56] J. S. Lee, J. L. O. Sheer, N. Lopez, and S. Rosenbaum, "Coverage of obesity treatment: a state-by-state analysis of Medicaid and state insurance laws," Public Health Reports, vol. 125, no. 4, pp. 596-604, 2010.

[57] R. Ross and A. J. Bradshaw, "The future of obesity reduction: beyond weight loss," Nature Reviews: Endocrinology, vol. 5, no. 6, pp. 319-325, 2009.

[58] C. Tudor-Locke, M. M. Brashear, W. D. Johnson, and P. T. Katzmarzyk, "Accelerometer profiles of physical activity and inactivity in normal weight, overweight, and obese U.S. men and women," International Journal of Behavioral Nutrition and Physical Activity, vol. 7, article 60, 2010.

[59] B. H. Hansen, I. Holme, S. A. Anderssen, and E. Kolle, "Patterns of objectively measured physical activity in normal weight, overweight, and obese individuals (20-85 years): a crosssectional study," PLoS ONE, vol. 8, no. 1, Article ID e53044, 2013. 


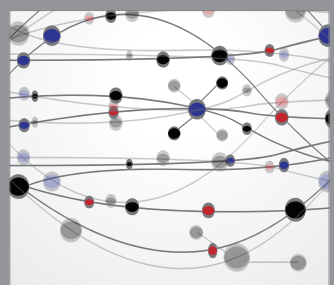

The Scientific World Journal
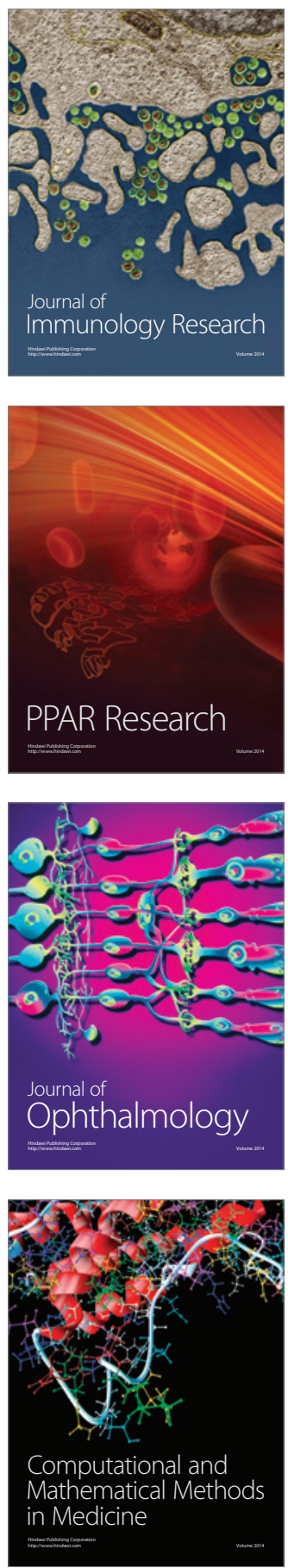

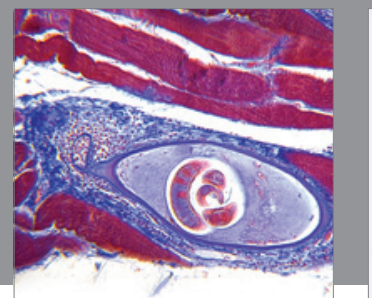

Gastroenterology

Research and Practice
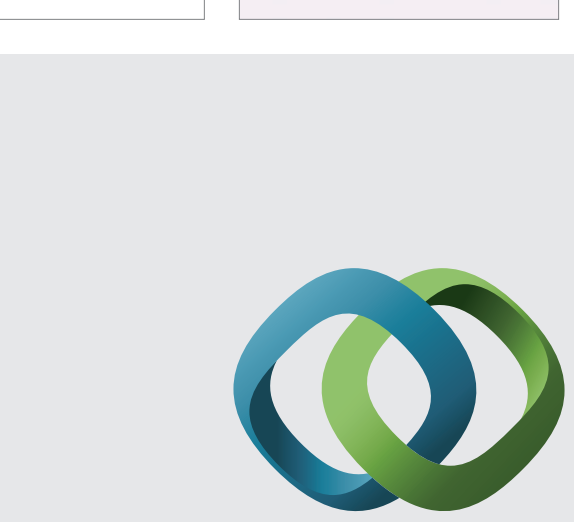

\section{Hindawi}

Submit your manuscripts at

http://www.hindawi.com
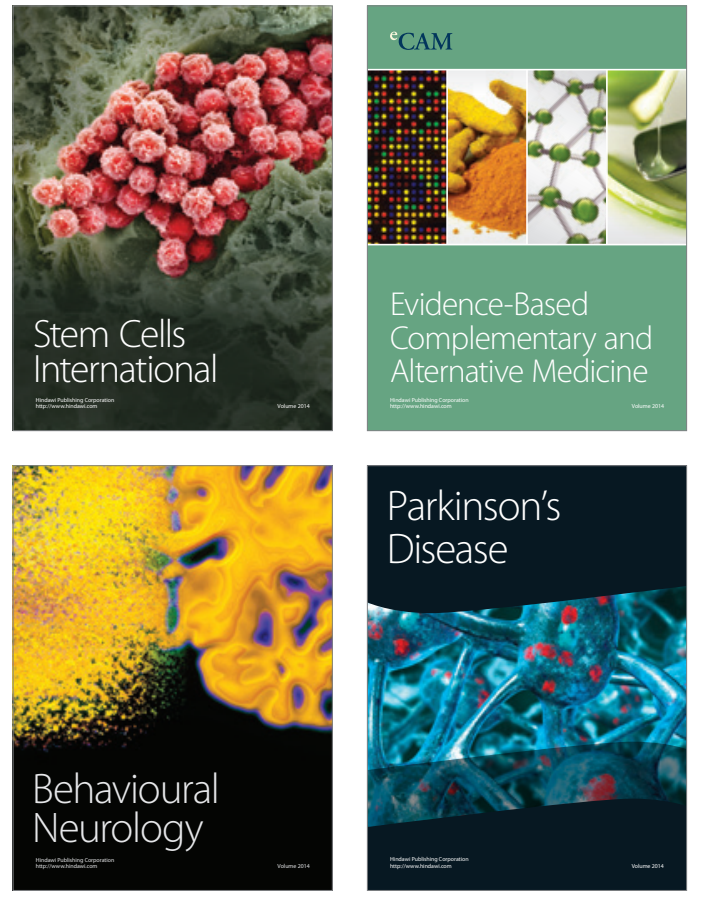
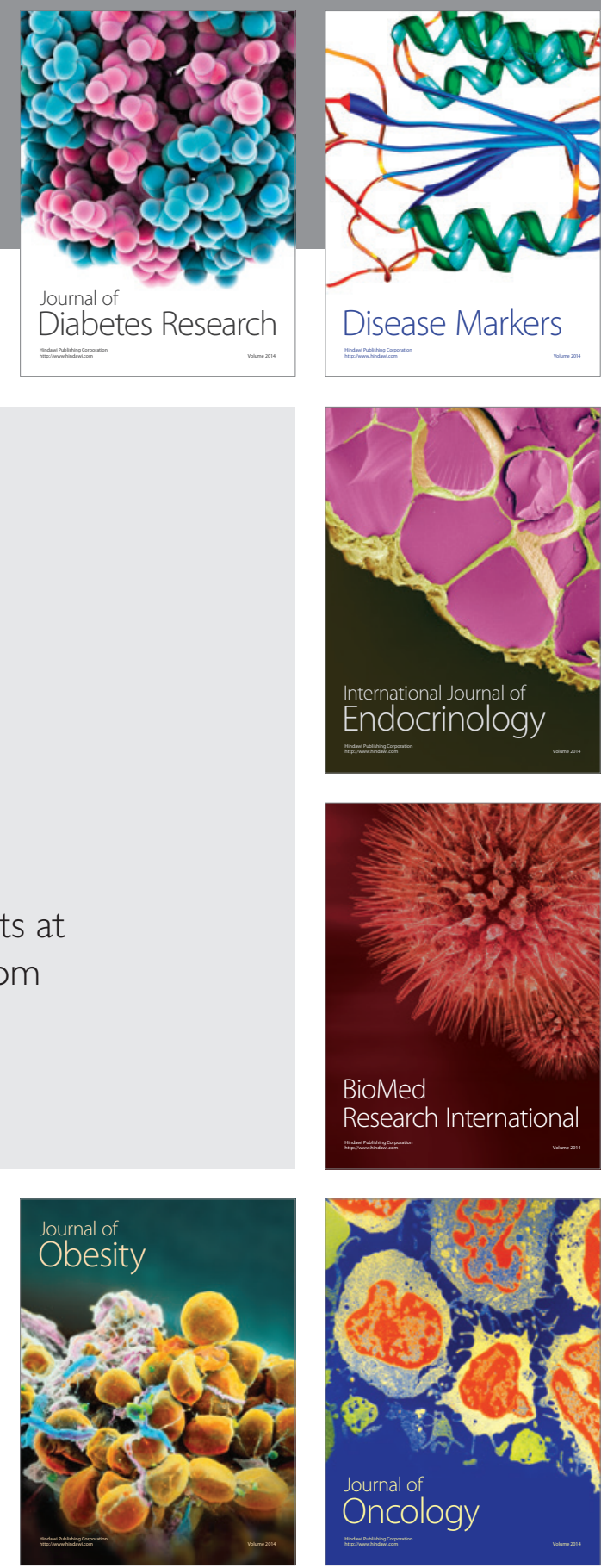

Disease Markers
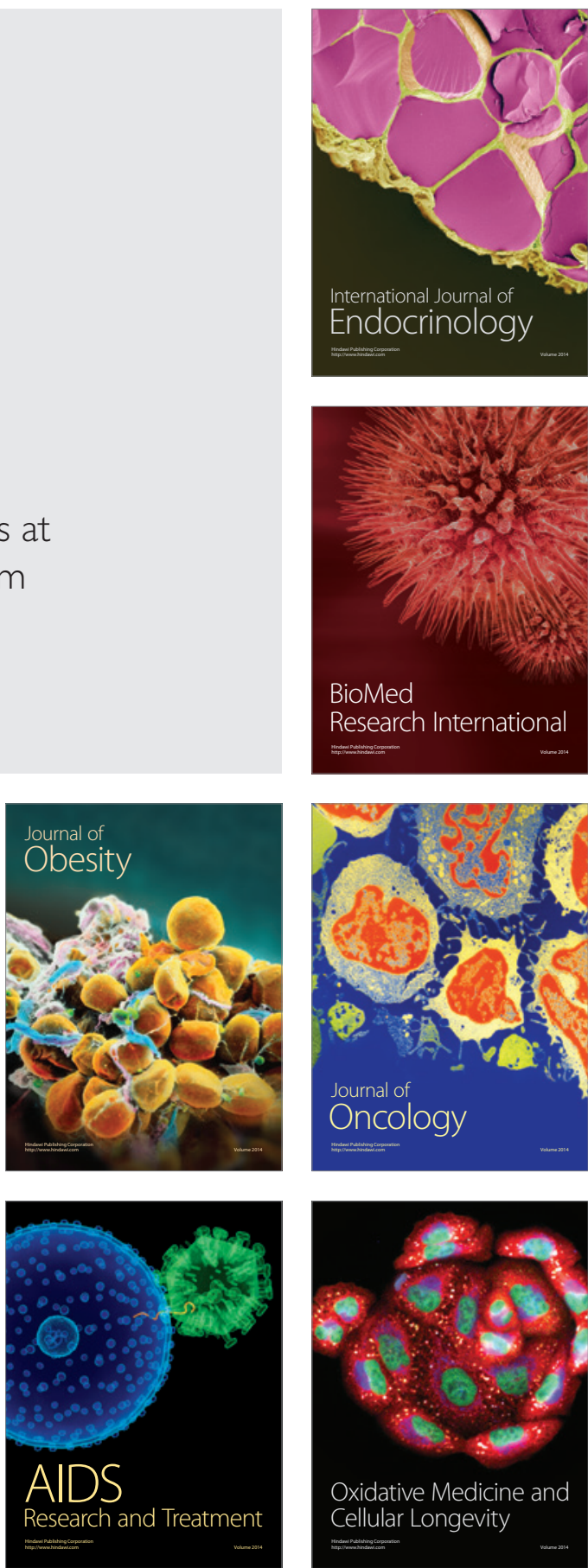